\title{
Esquema de Sincronização Projetiva Baseado em Caos e Análise de Lyapunov
}

\author{
Kevin H. M. Gularte*. Victor V. Graciano* Willian A. M. Gabalan** \\ José A. R. Vargas*
}

\author{
*Departamento de Engenharia Elétrica \\ **Departamento de Engenharia Mecânica \\ Universidade de Brasília \\ 70910-900 Campus Universitário Darcy Ribeiro, Brasília, DF, Brasil (e-mails: \\ kevinhmg@gmail.com,victorvelosog@gmail.com,wallanmolano@gmail.com,vargas@unb.br)
}

\begin{abstract}
This paper proposes a scheme for secure telecommunication based on projective synchronization of a chaotic Chen system, Lyapunov analysis, and analog electronics. Unlike most of the schemes usually found in the literature, the proposed scheme only requires the control action in one state equation of the slave system. The proposed scheme is robust against disturbances (internal and external) and simple, which is important since it leads to significant cost reductions when implemented employing analog electronics. Computational simulations using MATLAB/SIMULINK were performed to validate the robustness and simplicity of the proposed scheme.
\end{abstract}

Resumo: Neste artigo se propõe um esquema para telecomunicação segura baseado na sincronização projetiva de um sistema caótico de Chen, análise de Lyapunov e eletrônica analógica. Ao contrário da maioria dos esquemas usualmente encontrados na literatura, o esquema proposto requer apenas que o controle atue em uma das equações de estado do sistema escravo. O esquema proposto tem como vantagens ser robusto contra perturbações (internas e externas) e ser simples, o que é importante, uma vez que leva a importantes reduções de custos quando implementado utilizando eletrônica analógica. Além disso, para um maior grau de liberdade, projetou-se uma sincronização projetiva do tipo idêntica. Para validar a robustez e simplicidade do esquema proposto, simulações computacionais usando MATLAB/SIMULINK foram realizadas.

Keywords: Lyapunov analysis; projective synchronization; analog chãos; secure communication; Chen system.

Palavras-chaves: Análise de Lyapunov; sincronização projetiva; caos analógico; comunicação segura; sistema de Chen.

\section{INTRODUÇÃO}

Meios de telecomunicação no geral estão presentes no mundo todo há mais de 140 anos. Contudo ainda é uma tarefa difícil fazer com que sinais sigilosos sejam transmitidos de modo seguro. Basicamente, objetiva-se a criptografia de informações com a utilização de circuitos caóticos. Com um circuito caótico transmissor (ou mestre) codifica-se o sinal (ou mensagem) e com outro circuito caótico receptor (ou escravo) recupera-se a mensagem. Já existem outras maneiras de se criptografar uma mensagem analógica na literatura atual (Feki, 2003), entretanto nenhuma delas com custo tão baixo quanto a anteriormente proposta já que se podem utilizar apenas componentes analógicos para a montagem dos circuitos caóticos.

Os outros métodos de sincronização propostos na literatura também possuem limitações já que para fazer a sincronização eles precisam controlar todas as dimensões do sistema caótico em questão, ou seja, adicionam um sinal de controle em cada uma das equações diferenciais. Outra limitação é a de que os algoritmos de sincronização não levam em conta distúrbios no sinal codificado que podem surgir devido às interferências de qualquer natureza. É de suma importância que distúrbios estejam previstos nas análises feitas já que componentes eletrônicos possuem incertezas (tolerância) em seus valores nominais.

Mais especificamente, em Tirandaz et al. (2017); Yan et al., (2006) e Zhang et al. (2014) são propostos esquemas para comunicação segura baseados na teoria de estabilidade de Lyapunov e em diferentes técnicas de controle não linear (Backstapping, modo deslizante, adaptativo, projetivo, etc). Todavia, a maioria dos trabalhos considera um controle com o mesmo número de dimensões do sistema caótico (ElDessoky et al., 2017; Li, 2007; Wu et al., 2007) e não consideram a presença de distúrbios na análise de estabilidade. A presença de distúrbios raramente é considerada, como por exemplo, em Yang e Zhu (2013), que prevê um distúrbio inerente aos multiplicadores de sinal, mas que torna a solução demasiadamente complexa, pois requer um observador para fazer o controle corretamente.

A sincronização projetiva é caracterizada pela sincronia proporcional dos estados do sistema mestre e os estados do sistema escravo, ou seja, o sinal no escravo acompanha o sinal no mestre em uma proporção definida pelo fator de escala $\alpha$. Os primeiros autores analisaram a sincronização projetiva em apenas sistemas parcialmente lineares e mais recentemente descreveu-se a possibilidade de realizar esse 
tipo de sincronização em uma classe geral de sistemas caóticos. $\mathrm{O}$ fator $\alpha$ define tipos diferentes de sincronização análogas à sincronização projetiva como a MPS (modified projective sincronization, em que $\alpha$ é uma matriz), FPS (function projective sincronization, em que $\alpha$ é uma função), sincronização idêntica em que $\alpha=1$ e sincronização antifase em que alfa é igual a $\alpha=-1$. Convém notar que a sincronização projetiva é importante pois permite compatibilizar a sincronização de sistemas caóticos que operam em diferentes magnitudes ao mesmo tempo que permite elevar a segurança da codificação. Alguns casos de sincronização projetiva podem ser encontrados nos seguintes trabalhos: Mainieri e Rehacek (1999), Li (2007), Yan e Li (2005). Contudo, ao contrário do esquema proposto neste trabalho, em Mainieri e Rehacek (1999) e Li (2007) não foi projetado um controle do tipo subatuado. No caso de Yan e Li (2005) há um projeto de controle subatuado, porém distúrbios não foram considerados no seu projeto $\mathrm{e}$ simulação.

O objetivo do trabalho é, portanto, propor um sistema capaz de tornar a comunicação segura, simples e que leve em conta distúrbios de qualquer natureza. Além disso, almeja-se que a sincronização seja genérica, isto é, projetiva. Utiliza-se para tal um sistema caótico de Chen (Li e Chen, 2004) para o qual se propõe um controle em apenas o segundo estado, o que facilita a implementação do sistema proposto. A teoria de estabilidade de Lyapunov (Ioannou e Sun, 1996) permite concluir que o esquema proposto é robusto na presença de distúrbios limitados para a concepção do sistema caótico receptor responsável por decodificar o sinal, ao contrário de Aguila-Camacho, et al. (2016) e Sun e Shen, (2016) que não consideraram distúrbios na análise.

\section{FORMULAÇÃO DO PROBLEMA}

Considere o seguinte sistema caótico (Li e Chen, 2004)

$$
\begin{aligned}
& \dot{x}=-a x+a y \\
& \dot{y}=b y-c x-x z+u \\
& \dot{z}=-f z+x y
\end{aligned}
$$

Sendo $a=35 ; b=28 ; c=7$ e $f=3$. Observe que se trata de um sistema de Chen, onde $x(t), y(t)$ e $z(t)$ são os estados do sistema e $a, b, c$ e $d$ são constantes reais. Com base em (1), considere os seguintes sistemas mestre e escravo perturbados:

$$
\begin{aligned}
& \dot{x}_{m}=-a x_{m}+a y_{m} \\
& \dot{y}_{m}=b y_{m}-c x_{m}-x_{m} z_{m} \\
& \dot{z}_{m}=-f z_{m}+x_{m} y_{m} \\
& \dot{x}_{s}=-a x_{s}+a y_{s}+h_{1}(t) \\
& \dot{y}_{s}=b y_{s}-c x_{s}-x_{s} z_{s}+h_{2}(t)+u \\
& \dot{z}_{s}=-f z_{s}+x_{s} y_{s}+h_{3}(t)
\end{aligned}
$$

em que $x_{m}, y_{m}$ e $z_{m}$ são os estados do sistema mestre, e $x_{s}, y_{s}$ e $z_{s}$ são os estados do sistema escravo, e $h_{1}, h_{2}$ e $h_{3}$ são distúrbios presentes no sistema escravo.
Objetiva-se a sincronização dos sistemas (2) e (3) em que o sistema escravo tenha somente um sinal de controle escalar, ou seja, atuando apenas em um estado.

Comentário 1: Uma vez que o sistema (1) é caótico, seu comportamento depende muito das condições iniciais de forma que o sistema é sensível a mudanças nestas condições iniciais. Por causa disso e também pelo fato de ter comportamento aperiódico, a sincronização de sistemas caóticos geralmente é considerada mais complicada do que se fosse realizada em outros sistemas dinâmicos.

Hipótese 1: Assume-se que os distúrbios são limitados. Mais especificamente

$$
\begin{aligned}
& \left|h_{1}(t)\right| \leq \bar{h}_{1} \\
& \left|h_{2}(t)\right| \leq \bar{h}_{2} \\
& \left|h_{3}(t)\right| \leq \bar{h}_{3}
\end{aligned}
$$

onde $\bar{h}_{1}, \bar{h}_{2}$ e $\bar{h}_{3}$ são constantes desconhecidas.

Comentário 2: O objetivo para se apresentar os sistemas (2) e (3) em que os distúrbios $h$ são explicitamente considerados, é para ressaltar que o esquema de sincronização estudado é válido mesmo que na presença de mudanças inesperadas em sistemas dinâmicos.

\section{EQUAÇÃO DO ERRO DE SINCRONIZAÇÃO E SINAL DE CONTROLE PROPOSTO}

Define-se a dinâmica dos erros de sincronização como sendo:

$$
\begin{aligned}
& \dot{e}_{1}=\dot{x}_{s}-\alpha \dot{x}_{m} \\
& \dot{e}_{2}=\dot{y}_{s}-\alpha \dot{y}_{m} \\
& \dot{e}_{3}=\dot{z}_{s}-\alpha \dot{z}_{m}
\end{aligned}
$$

Substituindo-se (2) e (3) em (5), obtém-se que:

$$
\begin{aligned}
\dot{e}_{1} & =-a e_{1}+a e_{2}+h_{1} \\
\dot{e}_{2} & =-\left(e_{1} e_{3}+\alpha e_{1} z_{m}+\alpha e_{3} x_{m}+\alpha^{2} x_{m} z_{m}\right. \\
& \left.-\alpha x_{m} z_{m}\right)+b e_{2}-c e_{1}+h_{2}+u \\
\dot{e}_{3} & =\left(e_{1} e_{2}+\alpha e_{1} y_{m}+\alpha e_{2} x_{m}+\alpha^{2} x_{m} y_{m}\right. \\
& \left.-\alpha x_{m} y_{m}\right)-f e_{3}+h_{3}
\end{aligned}
$$

Uma vez definida a dinâmica dos erros, para que o sistema escravo sincronize corretamente com o sistema mestre, é necessário um sinal de controle $u$ adequado.

\section{Teorema 1:}

Considere os sistemas mestre e escravo descritos em (2) e (3) e a seguinte lei de controle proporcional com ganho variável

$$
u=-\psi e_{2}
$$

Deste modo, o erro de sincronização converge globalmente e em tempo finito para o conjunto compacto $\Omega=\left\{e \in \mathfrak{R}^{3} \mid\|e\| \leq \theta\right\}$, em que $\psi$ é uma constante positiva definida pelo usuário.

\section{Prova:}

Considere a seguinte candidata de Lyapunov 


$$
V=\frac{1}{2}\left(\gamma e_{1}^{2}+e_{2}^{2}+e_{3}^{2}\right)
$$

onde $\gamma>2$. Derivando (8) em relação ao tempo ao longo das trajetórias dos erros resulta

$$
\dot{V}=\gamma e_{1} \dot{e}_{1}+e_{2} \dot{e}_{2}+e_{3} \dot{e}_{3}
$$

Substituindo-se (6) em (9), tem-se

$$
\begin{aligned}
\dot{V} & =\gamma e_{1}\left(-a e_{1}+a e_{2}+h_{1}\right)+e_{2}\left[b e_{2}-c e_{1}\right. \\
& -\left(e_{1} e_{3}+\alpha e_{1} z_{m}+\alpha e_{3} x_{m}+\alpha^{2} x_{m} z_{m}-\alpha x_{m} z_{m}\right) \\
& \left.+h_{2}+u\right]+e_{3}\left[-f e_{3}+e_{1} e_{2}+\alpha e_{1} y_{m}+\alpha e_{2} x_{m}\right. \\
& \left.+\alpha^{2} x_{m} y_{m}-\alpha x_{m} y_{m}+h_{3}\right]
\end{aligned}
$$

Substituindo-se (7) em (10), obtém-se

$$
\begin{aligned}
& \dot{V}=-a \gamma e_{1}^{2}-e_{2}^{2}(\psi-b)-f e_{3}^{2}+\gamma e_{1} h_{1}+e_{2} h_{2} \\
& \quad+e_{3} h_{3}+e_{1} e_{2}(a \gamma-c)-\alpha e_{1} e_{2} z_{m}+\alpha e_{1} e_{3} y_{m} \\
& -\alpha^{2} e_{2} x_{m} z_{m}+\alpha^{2} e_{3} x_{m} y_{m}+\alpha e_{2} x_{m} z_{m}-\alpha e_{3} x_{m} y_{m}
\end{aligned}
$$

A partir de (4), observe que

$$
\begin{aligned}
-e_{2}\left(e_{1} \alpha z_{m}\right) & \leq 0,5 e_{1}^{2}(\alpha \bar{z})^{2}+0,5 e_{2}^{2} \\
e_{3}\left(e_{1} \alpha y_{m}\right) & \leq 0,5 e_{1}^{2}(\alpha \bar{y})^{2}+0,5 e_{3}^{2} \\
e_{2}\left[e_{1}(a \gamma-c)\right] & \leq 0,5 e_{1}^{2}(a \gamma-c)^{2}+0,5 e_{2}^{2} \\
-e_{2} \alpha^{2} x_{m} z_{m} & \leq 0,5 e_{2}^{2}+0,5\left(\alpha^{2} \bar{x} \bar{z}\right)^{2} \\
e_{3} \alpha^{2} x_{m} y_{m} & \leq 0,5 e_{3}^{2}+0,5\left(\alpha^{2} \bar{x} \bar{y}\right)^{2} \\
e_{2} \alpha x_{m} z_{m} & \leq 0,5 e_{2}^{2}+0,5(\alpha \bar{x} \bar{z})^{2} \\
-e_{3} \alpha x_{m} y_{m} & \leq 0,5 e_{3}^{2}+0,5(\alpha \bar{x} \bar{y})^{2} \\
e_{1}\left(\gamma h_{1}\right) & \leq 0,5\left(e_{1}^{2}+\gamma^{2} \bar{h}_{1}^{2}\right) \\
e_{2} h_{2} & \leq 0,5\left(e_{2}^{2}+\bar{h}_{2}^{2}\right) \\
e_{3} h_{3} & \leq 0,5\left(e_{3}^{2}+\bar{h}_{3}^{2}\right)
\end{aligned}
$$

Os termos $\bar{x}, \bar{y}$ e $\bar{z}$ são limitantes dos estados do sistema mestre, afinal se pressupõe que a planta original (o sistema mestre) é um sistema caótico limitado. Passando-se (11) para inequação e utilizando-se (12), advém

$$
\begin{aligned}
\dot{V} & \leq-e_{1}^{2}\left[a \gamma-0,5 \alpha^{2}\left(\bar{y}^{2}+\bar{z}^{2}\right)-0,5(a \gamma-c)^{2}\right. \\
& -0,5]-e_{2}^{2}(\psi-b-2,5)-e_{3}^{2}(f-2) \\
& +\left[0,5 \bar{x}^{2}\left(\bar{y}^{2}+\bar{z}^{2}\right)\left(\alpha^{4}+\alpha^{2}\right)+0,5\left(\gamma \bar{h}_{1}^{2}+\bar{h}_{2}^{2}+\bar{h}_{3}^{2}\right)\right]
\end{aligned}
$$

\section{Considere que}

$$
\begin{aligned}
\rho_{1} & =a \gamma-0,5 \alpha^{2}\left(\bar{y}^{2}+\bar{z}^{2}\right)-0,5(a \gamma-c)^{2}-0,5 \\
\rho_{2} & =\psi-b-2,5 \\
\rho_{3} & =f-2 \\
\beta & =0,5 \bar{x}^{2}\left(\bar{y}^{2}+\bar{z}^{2}\right)\left(\alpha^{4}+\alpha^{2}\right)+0,5\left(\gamma \bar{h}_{1}^{2}+\bar{h}_{2}^{2}+\bar{h}_{3}^{2}\right)
\end{aligned}
$$

Substituindo (14) em (13), tem-se

$$
\dot{V} \leq-e_{1}^{2} \rho_{1}-e_{2}^{2} \rho_{2}-e_{3}^{2} \rho_{3}+\beta
$$

Note que $\rho_{3}=1$ e que como $\gamma$ e $\psi$ são arbitrários, é possível defini-los de modo que $\rho_{1}$ e $\rho_{2}$ sejam maiores que $\rho_{3}$. Assim, (15) pode ser escrita como

$$
\dot{V} \leq-\|e\|^{2}+\beta
$$

Porém note que, pode-se dizer que $\alpha \mathrm{V}=e_{1}^{2}+(\alpha-1)\left(e_{2}^{2}+\right.$ $e_{3}^{2}$ ), sendo $\alpha=0,5 \gamma$, assim

$$
\dot{V} \leq-\alpha \mathrm{V}+\beta
$$

A inequação (17) implica

$$
t \leq \frac{1}{\alpha} \ln \left(\frac{1}{\alpha V-\beta}\right)
$$

Deste modo em (16), $\dot{V} \leq 0$ quando $\|e\|>\sqrt{\beta}:=\theta$. Como $\theta$ é constante, pode-se afirmar que o erro de sincronização é limitado. Definindo o conjunto compacto $\Omega=\left\{e \in \mathfrak{R}^{3} \mid\|e\| \leq \theta\right\}$, então se pode afirmar que se por qualquer razão $\|e\|$ deixar o conjunto residual $\Omega, \dot{V}$ se torna negativo definido e força a convergência do erro de sincronização para o conjunto residual $\Omega$, conforme (16). Ou seja, se a $\dot{V} \leq 0$ for satisfeita, a norma do erro somente poderá diminuir com o decorrer do tempo. Conclui-se dessa maneira que o erro de sincronização é limitado e converge para uma bola com raio igual a $\theta$. Observe em (18) que a limitação do erro ocorre em tempo finito.

Comentário 4: É importante notar que a constante $\beta$ poderia ser diminuído na prova caso o valor de $\psi$ for maior, ou seja, o valor $\psi$ pode parcialmente influenciar o valor de $\beta$ e consequentemente o valor de o valor de $\theta$. Desse modo a partir da escolha de parâmetros de projeto do controlador pode se levar a um erro de sincronização próximo de zero, mesmo que na presença de distúrbios limitados.

Comentário 5: Convém ressaltar que o esquema proposto, ao contrário da abordagem precursora (Cuomo et al., 1993), permite o ajuste arbitrário da velocidade da sincronização, o que pode ser feito através do parâmetro $\psi$. Adicionalmente, o algoritmo proposto considera a presença de distúrbios em todos os estados, o que, até onde os autores conhecem, normalmente não é considerado na literatura.

\section{SIMULAÇÃO}

Para validar o esquema de sincronização decidiu-se fazer simulações computacionais para o sistema (1). Utilizou-se para isso o software MATLAB/Simulink com o método ode113 com passo variável. Foi considerado como condições iniciais no sistema mestre que $x_{m}(0)=\left[\begin{array}{lll}1,5 & 2 & 5\end{array}\right] \mathrm{e}$ no sistema escravo que $x_{s}(0)=\left[\begin{array}{lll}0,1 & 0,3 & -0,1\end{array}\right]$. Para sincronização do sistema mestre e o escravo utilizou-se a lei de controle (7).

Escolheram-se os parâmetros $\alpha$ e $\psi$ como sendo $\alpha=1$ e $\psi=100$. Os distúrbios considerados foram $h_{1}=$ $0.5 \cos (6 t), h_{2}=3 \cos (10 t)$ e $h_{2}=\operatorname{sen}(4 t)$. Simulou-se a codificação e restauração de uma mensagem para que se pudesse analisar a eficiência e a robustez do canal sigiloso de comunicação proposto. A mensagem criptografada é uma soma de senos, $4 \operatorname{sen}(2 t)+\operatorname{sen}(10 t)$, concatenados ao primeiro estado.

As Figuras 1-6 mostram os resultados da sincronização 
obtida no MATLAB/Simulink. Percebe-se que há na Figura 1 uma discrepância entre os sinais no mestre e no escravo, isso é esperado uma vez que o sistema escravo sincroniza com o estado mestre sem a mensagem. Como a Figura 1 mostra o sistema mestre com a mensagem, a discrepância mostrada nessa figura é a própria mensagem. Nas Figuras 2 e 3 mostrase a sincronização dos outros estados, como não há mensagem sendo transmitida nesses estados, a sincronização, como esperado, teve um desempenho satisfatório, com erros de sincronização muito pequenos. Na Figura 4 pode-se se observar a comparação entre a mensagem codificada (sinal do estado $x_{m}$ acrescentado da mensagem) e a mensagem original (aquela que se quer transmitir). A Figura 5 mostra a mensagem original e a mensagem recuperada. A mensagem recuperada é obtida a partir da diferença entre $x_{s}$ e a mensagem codificada. Como esperado a diferença entre as duas mensagens é muito pequena, demonstrando o correto funcionamento do sincronizador. A Figura 6 mostra essa diferença de maneira mais precisa. Novamente se observa que o erro é pequeno sendo esse o resultado desejado. Em outras palavras, a mensagem foi recuperada por meio do receptor de maneira satisfatória.

Note que apesar do controle projetivo só estar presente somente no segundo estado $y$ e que há distúrbios em todos os estados, mesmo assim o primeiro e terceiro estados apresentaram desempenho satisfatório em termos de sincronização. Além disso, a mensagem no estado $x$ foi corretamente decodificada. Note que se precisasse de um resultado ainda melhor, é possível melhorar o processo de reconstrução da mensagem aumentando o valor do parâmetro $\psi$. Também é possível escalonar o sistema no tempo para assegurar uma sincronização mais rápida de forma que o $\psi$ não apresente um valor muito alto.

\section{CONCLUSÃO}

Neste artigo foi proposto um algoritmo de sincronização projetiva com base na teoria de estabilidade de Lyapunov para um sistema Chen sujeito a distúrbios limitados. Foi provado e validado via simulações que é necessário o emprego do sinal de controle em apenas um dos estados para realizar a sincronização completa dos sistemas mestre e escravo. Concluiu-se também a eficácia do método proposto para codificar e restaurar a mensagem tanto teoricamente quanto nas simulações.

\section{REFERÊNCIAS}

Aguila-Camacho, N., Duarte-mermoud, M. A., DelgadoAguilera, E. (2016). Adaptive synchronization of fractional Lorenz systems using a reduced number of control signals and parameters. Chaos, Solitons \& Fractals, 87, 1-11.

Cuomo, K. M., Oppenheim, A. V., Strogatz S. H. (1993). Synchronization of Lorenz-based chaotic circuits with applications to communications. IEEE Transactions on circuits and systems II: Analog and digital signal processing, 40(10), 626-633.
El-Dessoky, M. M., Alzahrany, E. O., Almohammadi, N. A. (2017). Function Projective Synchronization for Four Scroll Attractor by Nonlinear Control. Applied Mathematical Sciences, 11, 1247-1259.

Feki, M. (2003). An adaptive chaos synchronization scheme applied to secure communication. Chaos, Solitons \& Fractals, 18, 141-148, 2003.

Ioannou, P. A. \& Sun, J. (2012). Robust adaptive control. New Jersey, USA: Prentice-Hall, Inc., Englewood Cliffs.

Li, C., \& Chen, G. (2004). Chaos in the fractional order Chen system and its control. Chaos, Solitons \& Fractals, 22(3), 549-554.

Li, G. (2007). Modified projective synchronization of chaotic system. Chaos, Solitons \& Fractals, 32, 1786-1790.

Mainieri, R., \& Rehacek, J. (1999). Projective synchronization in three-dimensional chaotic systems. Physical Review Letters, 82(15), 3042.

Sun, J., \& Shen, Y. (2016). Adaptive anti-synchronization of chaotic complex systems and chaotic real systems with unknown parameters. Journal of Vibration and Control, 22, 2992-3003.

Tirandaz, H., Ahmadnia M., Tavakoli, H. (2017). Adaptive Projective Lag Synchronization of $\mathrm{T}$ and $\mathrm{Lu}$ Chaotic Systems. International Journal of Electrical and Computer Engineering (IJECE), 7, 3446-3453.

Wu, X., Chen, G., Cai, J. (2007). Chaos synchronization of the master-slave generalized Lorenz systems via linear state error feedback control. Physica D: Nonlinear Phenomena, 229, 52-80.

Yan, J., Hung, M., Chiang, T., Yang, Y. (2006). Robust synchronization of chaotic systems via adaptive sliding mode control. Physics Letters A, 356, 220-225.

Yan, J., \& Li, C. (2005). Generalized projective synchronization of a unified chaotic system. Chaos, Solitons \& Fractals, 26(4), 1119-1124.

Yang, J., \& Zhu, F. (2013). Synchronization for chaotic systems and chaos-based secure communications via both reduced-order and step-by-step sliding mode observers. Communications in Nonlinear Science and Numerical Simulation, 18, 926-937.

Zhang, J., Li, C., Zhang, H., Yu, J. (2004). Chaos synchronization using single variable feedback based on backstepping method. Chaos, Solitons \& Fractals, 21, 1183-1193. 


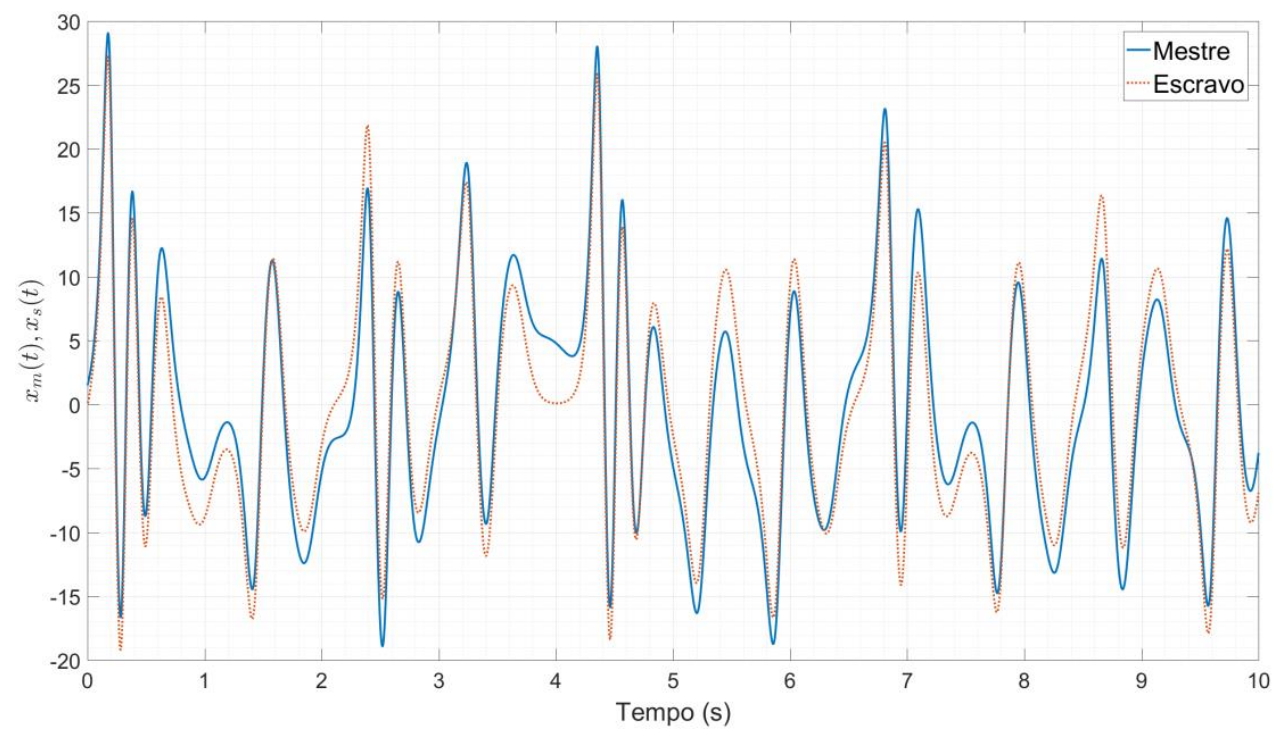

Fig. 1 Desempenho na sincronização de $x_{m}(t)$ e $x_{s}(t)$

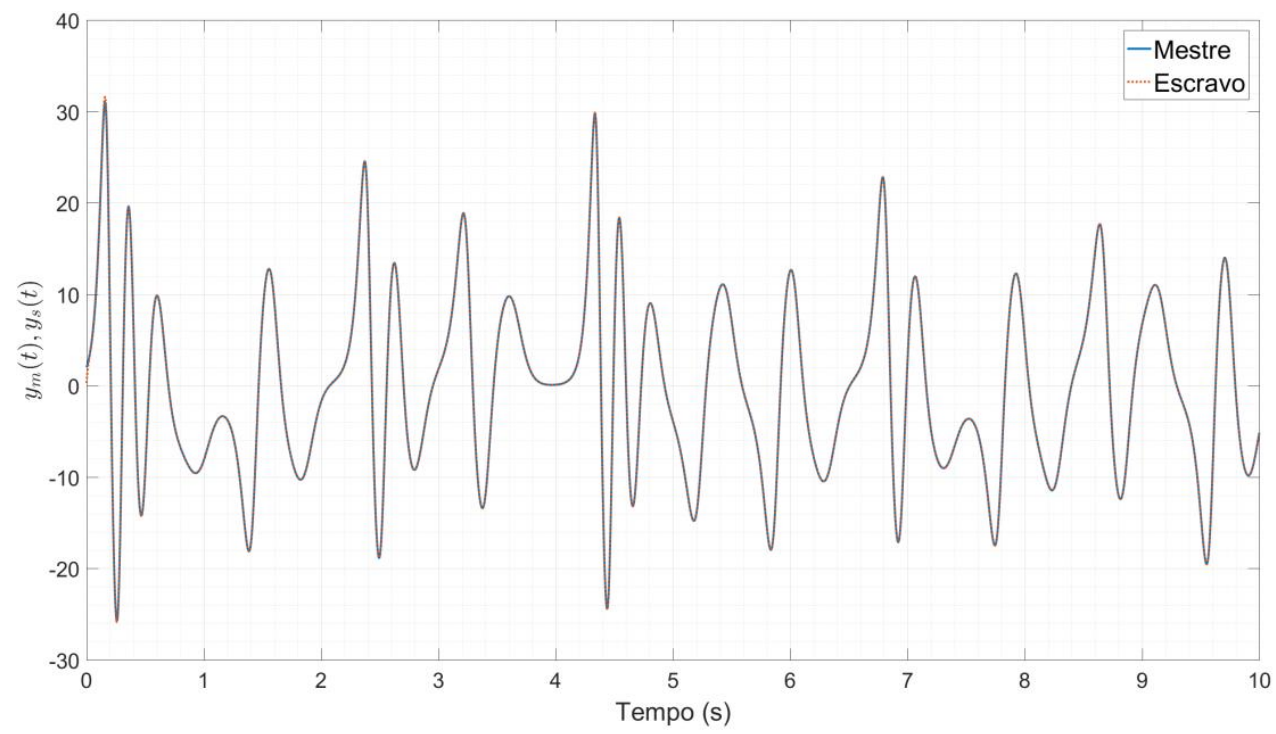

Fig. 2 Desempenho na sincronização de $y(t)$ e $y_{s}(t)$

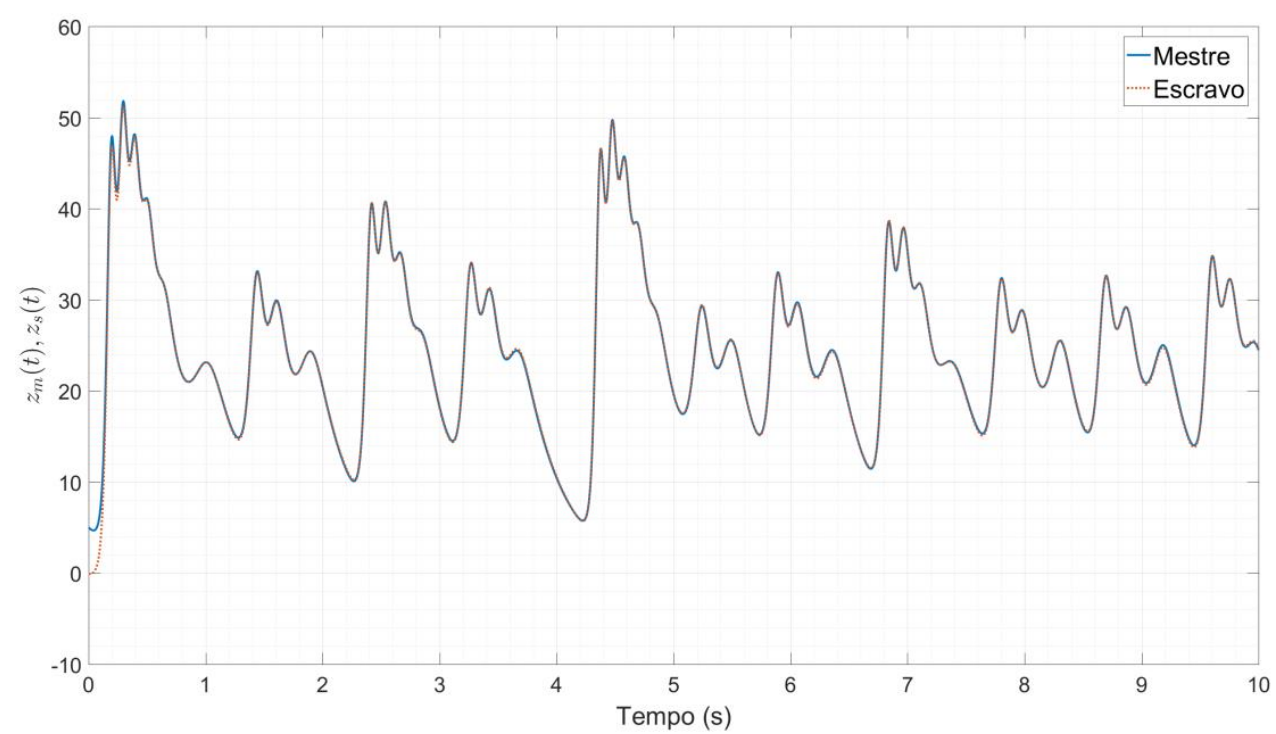

Fig. 3 Desempenho na sincronização de $z_{m}(t)$ e $z_{s}(t)$ 


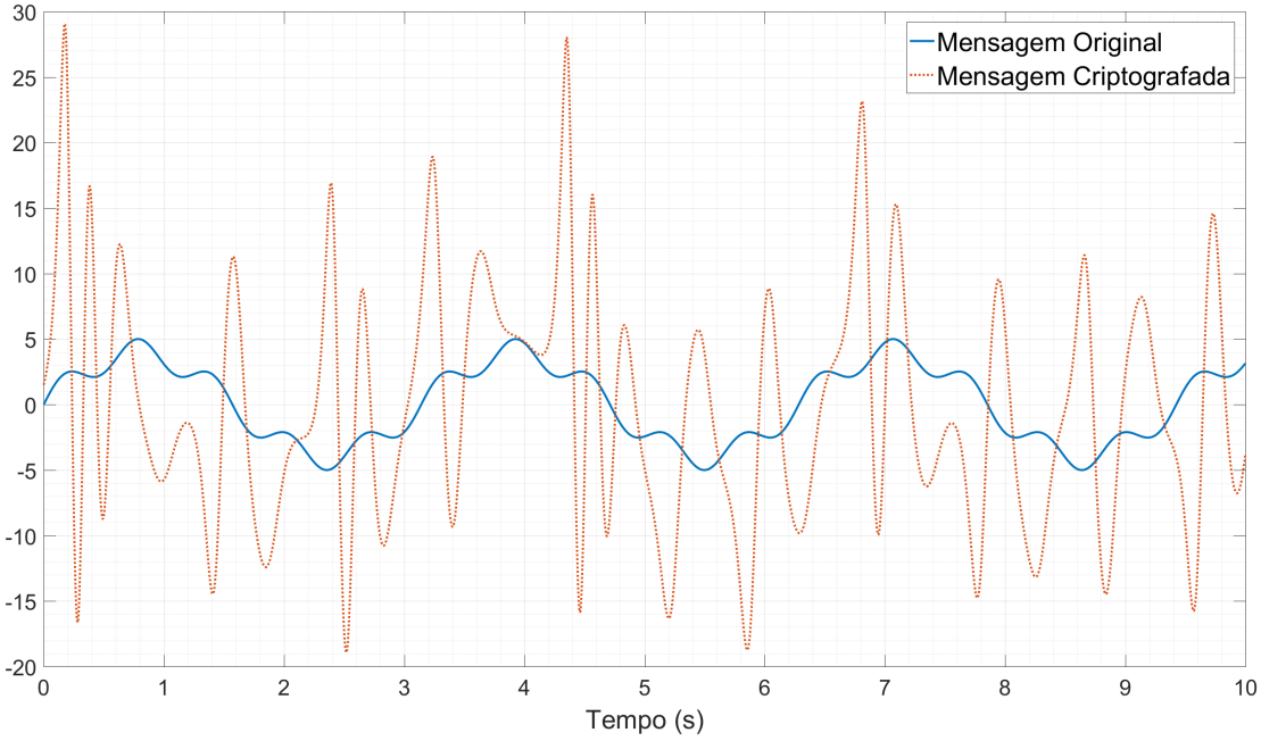

Fig. 4 Mensagem original e mensagem criptografada

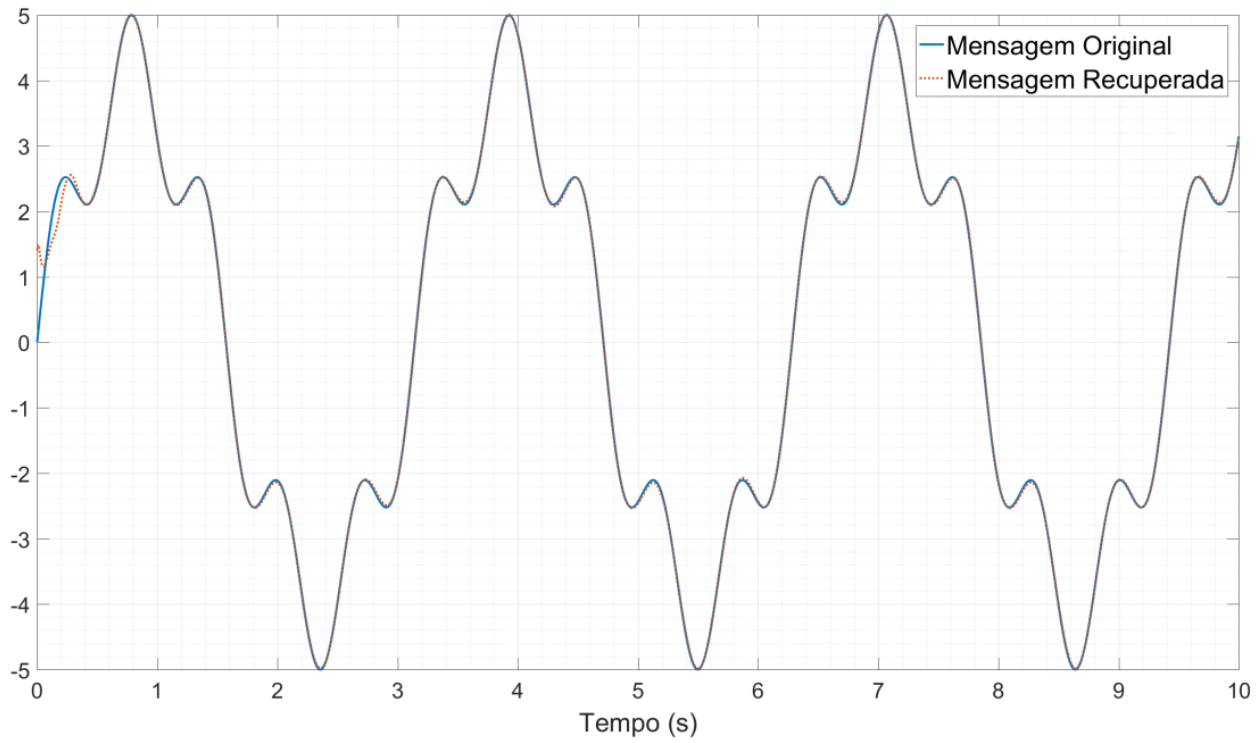

Fig. 5 Mensagem original e mensagem recuperada

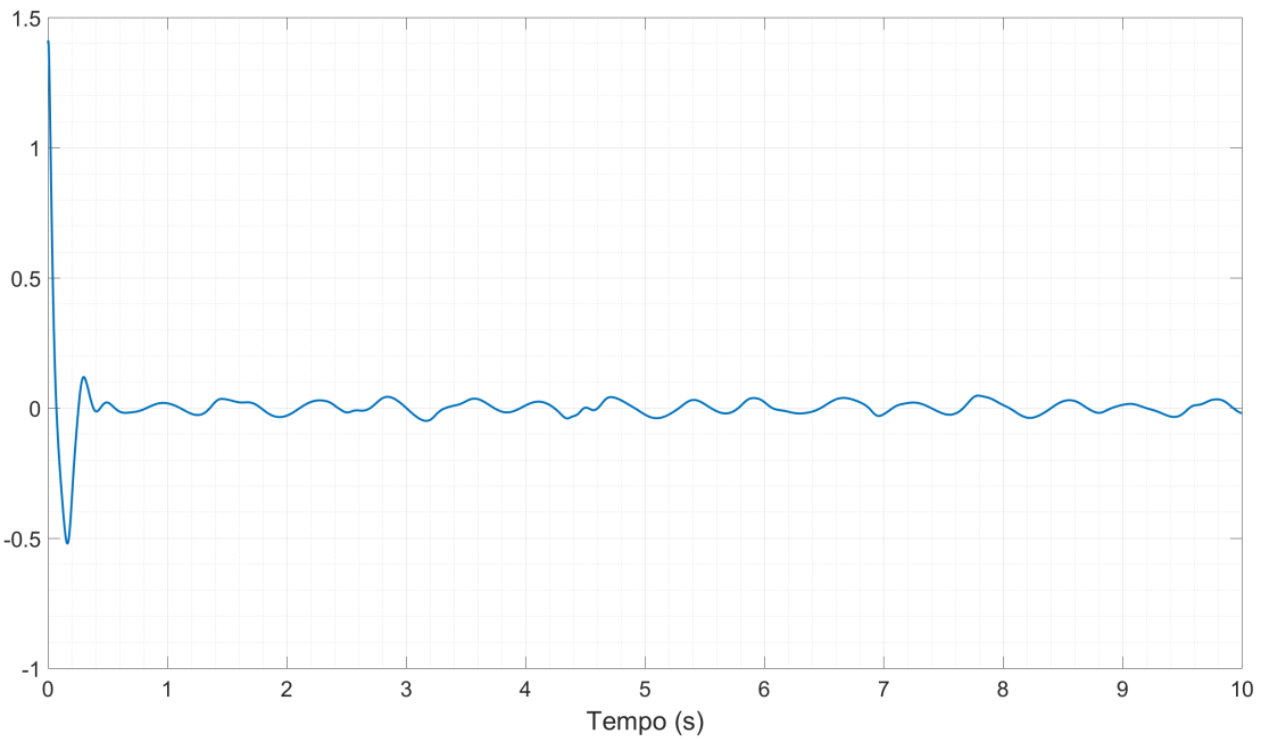

Fig. 6 Diferença entre a mensagem recuperada e a mensagem original 